\title{
Corrections
}

\section{Correction: Adnani et al., Zac1 Regulates the Differentiation and Migration of Neocortical Neurons via Pac1}

In the article "Zac1 Regulates the Differentiation and Migration of Neocortical Neurons via Pac1" by Lata Adnani, ${ }^{1,4,5 *}$ Lisa Marie Langevin, ${ }^{1,4,5 \star}$ Elodie Gautier, ${ }^{6}$ Rajiv Dixit, ${ }^{1,4,5}$ Kari Parsons, ${ }^{2,5}$ Saiqun Li, ${ }^{1,4,5}$ Gaurav Kaushik, ${ }^{1,4,5}$ Grey Wilkinson, ${ }^{1,4,5}$ Richard Wilson, ${ }^{3,4,5}$ Sarah Childs, ${ }^{1,4}$ Minh Dang Nguyen, ${ }^{2,5}$ Laurent Journot, ${ }^{7}$ Colette Dehay, ${ }^{6}$ and Carol Schuurmans, ${ }^{1,4,5}$ which appeared on pages 13430-13447 of the September 30, 2015 issue, the affiliations for Drs. Laurent Journot and Elodie Gautier and Colette Dehay were inadvertently reversed. Their corrected affiliations are: Elodie Gautier ${ }^{6,7}$ and Colette Dehay ${ }^{6,7}$ : ${ }^{6}$ Stem Cell and Brain Research Institute, INSERM U846, 69500, Bron, France, and ${ }^{7}$ University of Lyon, University of Lyon I, 69003, Lyon, France; and Laurent Journot $^{8}$ : Institut de Génomique Fonctionnelle, UMR5203 CNRS — U661 INSERM — Université de Montpellier, 34094 Montpellier Cedex 05 France. This error does not affect data interpretations or conclusions. It has been corrected in the online PDF version.

DOI:10.1523/JNEUROSCI.3858-15.2015

\section{Correction: Pifl et al., Is Parkinson's Disease a Vesicular Dopamine Storage Disorder? Evidence from a Study in Isolated Synaptic Vesicles of Human and Nonhuman Primate Striatum}

In the article “Is Parkinson's Disease a Vesicular Dopamine Storage Disorder? Evidence from a Study in Isolated Synaptic Vesicles of Human and Nonhuman Primate Striatum” by Christian Pifl, Alex Rajput, Harald Reither, Javier Blesa, Carmen Cavada, José A. Obeso, Ali H. Rajput, and Oleh Hornykiewicz, which appeared on pages 8210-8218 of the June 11, 2014 issue, a couple of errors were discovered and are corrected below.

In the Materials and Methods section on page 8212, left column, first paragraph below Table 2, line 6, the function in the sentence listed as "25 $\mu \mathrm{l} 4 \mathrm{~nm}( \pm)$-a-dihydrotetrabenazine $\left[2-{ }^{3} \mathrm{H}\right]$ ” should be corrected to read as "25 $\mu \mathrm{l} 12 \mathrm{~nm}( \pm)$-a-dihydrotetrabenazine $\left[2-{ }^{3} \mathrm{H}\right]$."

In the Discussion section on page 8216, right column, the first word in the legend for Figure 5 needs to be changed from "Protein" to "Proton." The corrected Figure 5 legend is listed below.

Figure 5. Proton gradient measurement on synaptic vesicles prepared from putamen of patients with PD and controls. Synaptic vesicle preparations ( $47 \pm 4$ and $48 \pm 7 \mu$ g protein for controls and $\mathrm{PD}$, respectively) were incubated with $2 \mu \mathrm{M}$ acridine orange and acidification was measured by quenching of fluorescence at $493 \mathrm{~nm}$ (excitation) and $530 \mathrm{~nm}$ (emission) after addition of $0.5 \mathrm{~mm}$ MgATP at $60 \mathrm{~s}$ and proton gradient abolition by addition of $1 \mu \mathrm{M}$ carbonyl cyanide FCCP at $300 \mathrm{~s}$. Shown are mean values of fluorescent traces shifted to 0 fluorescence at the time interval 0-58 \pm SE (gray area) of four controls $(\boldsymbol{A})$ and six PD patients $(\boldsymbol{B})$.

These corrections do not affect the main conclusions and interpretations of the paper. They have been corrected in the online PDF version.

DOI: $10.1523 / J N E U R O S C I .3865-15.2015$ 